and, therefore, the magnetic field is uniform in the film and equal to the applied magnetic field.

${ }^{5}$ One expects that the size of the superconducting "islands" must be at least as large as the superconducting coherence length. The thin films studied here are type-II superconductors and the coherence length $\xi$ is determined from the relation $\xi=\frac{1}{3}\left(l v_{\mathrm{F}} \hbar / \Delta\right)^{1 / 2}$, where $l$ is the mean free path, $v_{\mathrm{F}}$ the velocity of the electrons at the Fermi surface, and $\Delta$ the energy gap [P. G. de Gennes (private communication)]. Using this relation we obtain $\xi \sim 200-400 \AA$ for the films studied. Since this is much shorter than the width of the strips, the "island" picture is feasible at least with respect to this consideration.

\title{
CONDUCTION BAND MINIMA IN AlAs AND AlSb
}

\author{
C. A. Mead \\ California Institute of Technology, Pasadena, California \\ and \\ W. G. Spitzer \\ Electrical Engineering Department, University of Southern California, Los Angeles, California \\ (Received 19 August 1963)
}

The photoresponse of surface barrier rectifiers ${ }^{1}$ made by evaporating a metal such as gold or platinum on a cleaved surface of AlAs and AlSb has been measured in the front wall configuration. The photoresponse of such units for $h \nu>E_{g}$, where $E_{g}$ is the energy gap, will be proportional to the absorption coefficient as long as the optical attenuation length is large compared to both the width of the space-charge region and the minority carrier diffusion length. The analysis is essentially the same as that for $p-n$ junctions with the exception that the barrier is at the surface and hence more sensitive to photons of high absorption coefficient. Photoinjection of carriers from the metal into the semiconductor for photon energies where $h \nu<E_{g}$ can also be observed. ${ }^{1}$

If for indirect band-to-band transitions ${ }^{2}$ the absorption coefficient $\alpha$ is proportional to $\left(h \nu-h \nu_{t}\right)^{2}$, where $h \nu_{t}$ is the threshold energy for the absorption and if the assumptions made above are valid, then the (photoresponse) ${ }^{1 / 2}$ should be proportional to $h \nu-h \nu_{t}$. For direct band-to-band transitions the absorption rises to large values much more rapidly near the direct threshold, and the photoresponse should also show an abrupt rise. As in the case of the absorption measurements, it can be anticipated that indirect processes which occur for higher energies than the direct one will be largely obscured. Photomeasurements of this type have been used to determine the dependence on composition of both the direct and indirect transitions in the $\mathrm{Ga}\left(\mathrm{As}_{1}-x_{x} P_{x}\right.$ ) system. ${ }^{3}$

The room-temperature energy gap ${ }^{4}$ of AlAs is apparently close to $2.2 \mathrm{eV}$. The value $2.16 \mathrm{eV}$ is frequently given, but the present authors were unable to locate the source of this value in the literature. Paul $^{5}$ has pointed out that systematic extrapolation from silicon would suggest that the deepest minima in the conduction band are in the (100) directions $\left(\Delta_{1}\right.$ states $)$. To the best of the authors' knowledge, no other experimental information concerning either the (100) minima or any of the higher lying minima in this material is available.

The room-temperature energy gap ${ }^{6,7}$ of $\mathrm{AlSb}$ is approximately $1.5 \mathrm{eV}$ and again extrapolation from silicon suggests (100) conduction-band minima. Pressure measurements ${ }^{8}$ and electron effective-mass studies ${ }^{9}$ tend to confirm this prediction. It has been suggested that the (000) minimum is $\sim 0.3 \mathrm{eV}$ above the (100) minima. This conclusion was drawn from optical and electrical measurements ${ }^{7}$ of the $\left(\mathrm{Ga}_{1-x} \mathrm{Al}_{x}\right) \mathrm{Sb}$ system and was supported by infrared measurements ${ }^{10}$ which showed an absorption band in $n$-type material with a threshold of $0.29 \mathrm{eV}$. The absorption was attributed to interband transitions between the two types of minima. However, this interpretation has been questioned recently ${ }^{5}$ for similar infrared evidence in the case of GaP.

The results of the measurements on AlAs are illustrated in Fig. 1(a), where the (photovoltage) $)^{1 / 2}$ vs $h \nu$ is plotted for gold on an $n$-type sample. Very similar results were obtained for several samples. The low-energy response, $h \nu<E_{g}$, arises from photoinjection from the metal. $\mathrm{Ex}-$ trapolation of this response to $h \nu>E_{g}$ and subtracting from the measured values shows clearly both the indirect and direct transitions. Again extrapolation of the indirect response and subtraction gives just the direct edge. The values of $h \nu_{t}$ are $2.1 \mathrm{eV}$ for the indirect process and 2.9 $\mathrm{eV}$ for the direct threshold. The results confirm the indirect nature of the absorption edge the $h \nu_{t}$ 


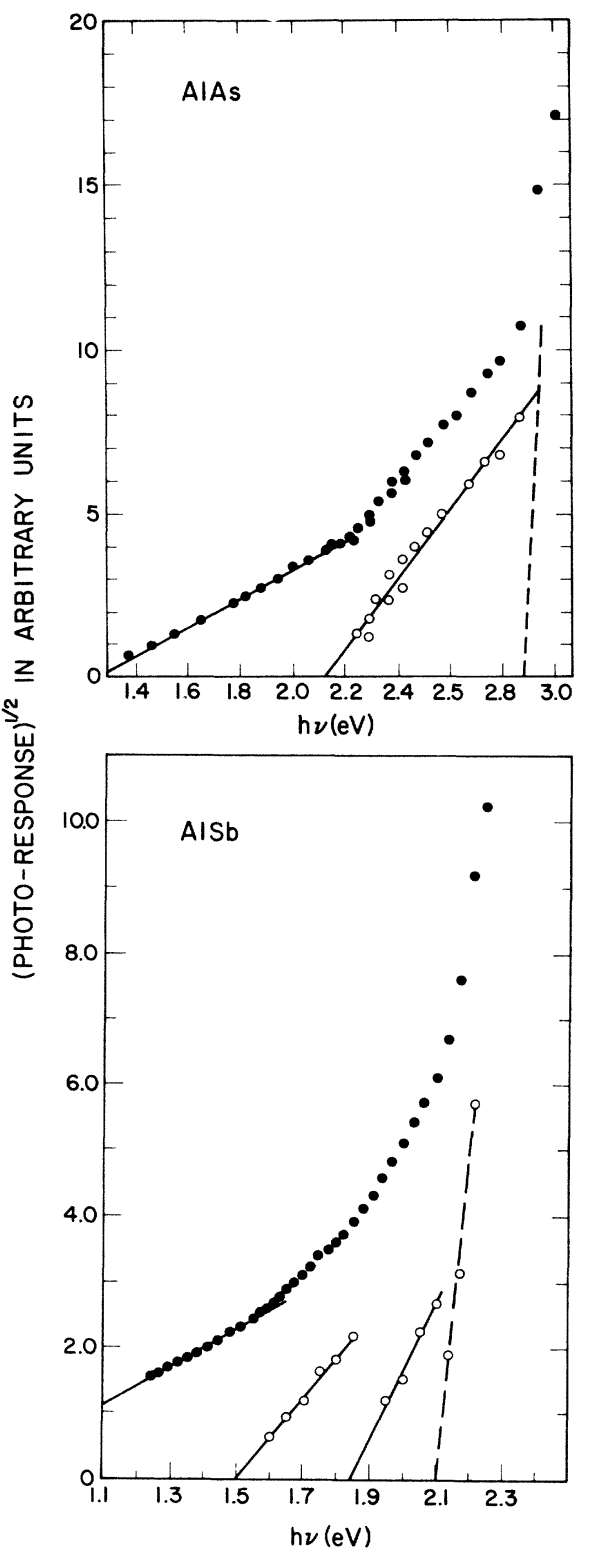

FIG. 1. Photovoltaic response of surface barrier contacts on AlAs and AlSb. The straight lines are the result of the subtraction procedure described in the text.

$=2.1 \mathrm{eV}$ is in reasonable agreement with the often quoted $E_{g}=2.16 \mathrm{eV}$ value. A dashed line is used for the direct transition response, since the rise is sufficiently abrupt that its dependence on $h \nu$ is not given by the data.

The photoresponse for $p$-type AlSb is shown in Fig. 1(b). Similar data were obtained for several samples. This material does not show breaks in slope as clearly as AlAs and other materials previously studied. It is clear, however, that at the low-energy end of the data where $h \nu<1.5 \mathrm{eV}$, the response from the metal is being observed.

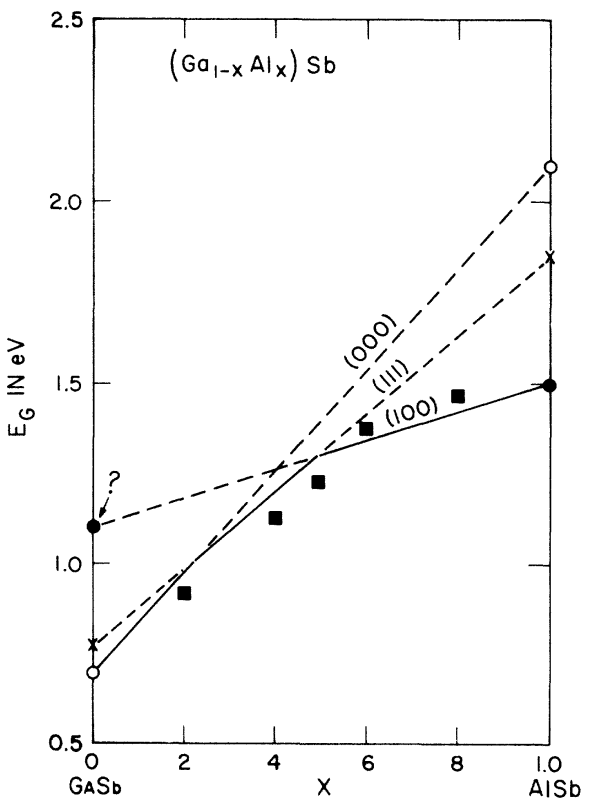

FIG. 2. Probable identification of conduction-band minima in ( $\mathrm{GaAl}) \mathrm{Sb}$ based upon the AlSb minima determined from Fig. 1. Square points are from reference 6 .

Only the high-energy portion of the metal response is shown in Fig. 1. It is also clear that the rapid rise corresponding to the direct transition does not occur at $1.85 \mathrm{eV}$ as suggested by Ehrenreich's extrapolation ${ }^{7}$ of the $\left(\mathrm{Ga}_{1}-x \mathrm{Al}_{x}\right) \mathrm{Sb}$ system but rather has a threshold in excess of $2 \mathrm{eV}$. If the subtraction process described previously is used, the data may be resolved into three different bandto-band transitions with threshold energies of $1.50,1.85$, and $2.10 \mathrm{eV}$. If it is assumed that the $\Delta_{1}$ states form the bottom of the conduction band, then the $1.85-\mathrm{eV}$ value must relate to transitions to the (111) or $L_{1}$ minima. These results suggest a reinterpretation of the $\left(\mathrm{Ga}_{1}-x \mathrm{Al}_{x}\right) \mathrm{Sb}$ data as shown in Fig. 2, where the separation of the $\overrightarrow{\mathrm{k}}=0$, (100), and (111) minima for $x=0$ were taken from the literature. ${ }^{11,12}$ The $(100)$ position is much less certain than that for the other minima. This interpretation suggests that the absorption edge is direct only for $x \leqq 0.2$. Unfortunately the absorption measurements are not presented in a manner such that direct and indirect transitions can be distinguished. It is clear that careful work is necessary before the conduction band of the solid solutions is well understood.

The authors are indebted to Derek Bolger of Standard Telecommunication Laboratories for supplying the AlAs, and H. M. Simpson for fabrication of the units. The work was supported in part by the U. S. Office of Naval Research and 
the International Telephone and Telegraph Corporation.

\footnotetext{
${ }^{1}$ C. R. Crowell, W. G. Spitzer, L. E. Howarth, and E. E. LaBate, Phys. Rev. 127, 2006 (1962); C. A. Mead and W. G. Spitzer, Appl. Phys. Letters 2, 74 (1963); W. G. Spitzer and C. A. Mead (to be published).

${ }^{2}$ T. S. Moss, Optical Properties of Semiconductors (Butterworths Scientific Publications, Ltd., London, 1959), 2nd ed., Chap. 3.

${ }^{3}$ W. G. Spitzer and C. A. Mead (to be published). ${ }^{4}$ Semiconductors, edited by N. B. Hannay (Reinhold Publishing Corporation, New York, 1959), Chap. 9, p. 417; C. Hilsum and A. C. Rose-Innes, Semiconducting III-V Compounds (Pergamon Press, New York, 1961), p. 3. R. Braunstein and E. O. Kane, J. Phys. Chem. Solids 23, 1423 (1962); R. Willardson and E. P.
}

Stambaugh, Compound Semiconductors, edited by R. K. Willardson and H. L. Goering (Reinhold Publishing Corporation, New York, 1962), Vol. 1, p. 186.

${ }^{5}$ W. Paul, J. Appl. Phys., Suppl. $\underline{32}, 2080$ S (1961).

${ }^{6}$ I. I. Burdigan, Fiz. Tverd. Tela $\underline{1}, 1360$ (1959)

[translation: Soviet Phys. - Solid State 1, 1246 (1960)].

${ }^{7}$ H. Ehrenreich, J. Appl. Phys., Suppl. 32, 2155S

(1961), Fig. 3 .

${ }^{8}$ A. L. Edwards and H. G. Drickamer, Phys. Rev. 122,1149 (1961).

${ }^{9}$ T. S. Moss, 1962 Semiconductor Conference (The Institute of Physics, London, 1962), p. 295.

${ }^{10} \mathrm{~W}$. J. Turner and W. E. Reese, Phys. Rev. 117 , $1103(1960)$.

${ }^{11}$ A. Sagar and R. C. Miller, J. Appl. Phys., Suppl. $32,2073 \mathrm{~S}$ (1961).

${ }^{12}$ W. M. Becker, A. K. Ramdas, and H. Y. Fan, ibid., 2094S (1961).

\title{
THERMOLUMINESCENCE OF THALLIUM-ACTIVATED ALKALI HALIDES
}

\author{
M. Schlesinger* and A. Halperin \\ Department of Physics, The Hebrew University of Jerusalem, Jerusalem, Israel \\ (Received 5 September 1963)
}

In a recent Letter Hersh and Hadley ${ }^{1}$ have compared the thermoluminescence and optical absorption of strained alkali halides to those of thalliumactivated crystals. From the similarities in behavior in both cases, they have concluded that (1) the trapping levels in thallium-activated crystals were characteristic of the undoped host crystals; (2) the emission in the main thermoluminescence glow peaks was due to the release of positive holes from $V_{k}$ centers and the excess energy might be released when a hole encounters an electron trapped at or near an impurity.

During the last few years we were occupied in this laboratory in an investigation on the optical properties of thermally treated alkali halide crystals. Comparison to the properties of thalliumdoped crystals proved interesting.

$\mathrm{We}^{2}$ as well as other authors ${ }^{3}$ fully agree with point one in Hersh and Hadley's conclusions. Our results, however, seem to disagree with the second conclusion of these authors as stated above. In other words, while our results imply that the main trapping levels in thallium-activated crystals are characteristic of the host crystal, their thermoluminescence emission spectrum seems to be characteristic of $\mathrm{Tl}^{+}$ions.

The crystals examined in our work were $\mathrm{NaCl}$, $\mathrm{KCl}, \mathrm{KBr}$, and $\mathrm{KI}$, and the results were essentially similar for all the four alkali halides. Figure 1 gives, for example, the glow curves for $\mathrm{KCl}$ crystals. "The dashed curve is for a "pure" crystal heat treated for $120 \mathrm{~min}$ at $600^{\circ} \mathrm{C}$, and the continuous one is for a crystal containing $0.1 \%$ thallium. The heating rate was $15 \% \mathrm{~min}$.

The similarity between the two curves, especially in the temperature range of $190-250^{\circ} \mathrm{K}$, is striking. Furthermore, the thermal activation energies were found to be the same for the corresponding glow peaks, and even the kinetics was found to be of the same order in both cases, namely, of first order.

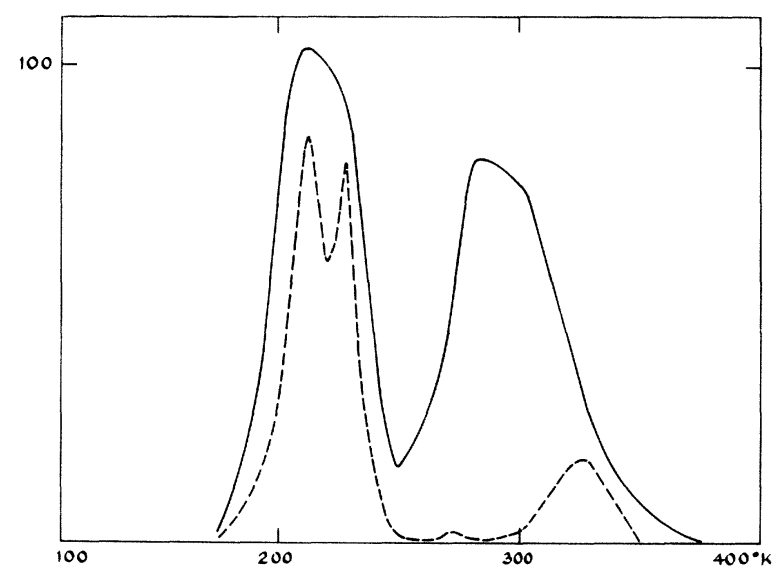

FIG. 1. The glow curves for $\mathrm{KCl}$ crystals. Dashed curve for a "pure" crystal heat treated for $120 \mathrm{~min}$ at $600^{\circ} \mathrm{C}$, continuous curve for a thallium-doped $(0.1 \%)$ on a three-times reduced scale. Both crystals were $\mathrm{x}$ rayed for $30 \mathrm{~min}$ at liquid nitrogen temperature. The heating rate was $15^{\circ} / \mathrm{min}$. 